\title{
Effects of Wall Reflection on the Per-Antenna Power Distribution of ZF-Precoded ULA for Indoor mmWave MU-MIMO Transmissions
}

\author{
Yixin Zhang, Graduate Student Member, IEEE, Jiliang Zhang, Senior Member, IEEE, \\ Xiaoli Chu, Senior Member, IEEE, Jie Zhang, Senior Member, IEEE
}

\begin{abstract}
Indoor access points (APs) with large-scale antenna arrays would commonly be deployed in the vicinity of a wall, where wall reflection (WR) affects the indoor electromagnetic (EM) wave propagation. In this paper, we investigate the effects of WR on the per-antenna power distribution of a transmit uniform linear array (ULA) adopting a zero-forcing (ZF) precoder. A new channel model is constructed to characterise the impact of both the line-of-sight (LOS) path and the WR path on indoor millimetre wave (mmWave) multi-user (MU) multiple-input multiple-output (MIMO) downlink transmissions. Specifically for the dual user equipment (UE) scenario, the $\mathrm{ZF}$ precoding matrix is analytically obtained and verified through simulations. The effects of WR on the per-antenna power distribution of the ZFprecoded ULA, in terms of the normalized power distribution and maximum power ratio (MPR), are evaluated through the comparisons between our proposed channel model and the pure LOS channel model. Our analytical and numerical results reveal the impact of AP configurations (the number of antennas and the AP-wall distance), multi-user spatial distribution (the angle of departure (AoD) and length of the LOS path for each user), and wall parameters (permittivity and thickness) on the power distribution across the ZF-precoded ULA. It is found that the effects of WR will exacerbate the uneven power distribution across the ZF-precoded ULA.
\end{abstract}

Index Terms-Wall reflection, power distribution, ZF precoding, ULA, indoor, mmWave, MU-MIMO, downlink.

\section{INTRODUCTION}

The combination of multiple-input multiple-output (MIMO) and millimetre wave (mmWave) technologies facilitates massive indoor high-rate wireless applications for future wireless networks by exploiting spatial multiplexing gains even in limited scattering environment [1]-[5]. While the deployment of indoor access point(s) (AP) would typically be optimised to maximise the throughput and/or coverage [6], customers may prefer to deploy the APs in positions that will not lead to inconveniencing usages of a room. A popular solution is to integrate the AP with an interior wall of the building [7]. In this sense, the interactions between the indoor electromagnetic wave (EM) propagation and the interior wall should not be neglected in the deployment of indoor APs [8], [9].

The EM wave bounced off an interior wall would have experienced multiple internal reflections, whose amplitudes and phases are totally changed [10]. The wall reflection (WR) is characterised by the reflection coefficient, which depends on the EM wave's polarisation, incidence angle, and the wall

Yixin Zhang, Jiliang Zhang, and Xiaoli Chu are with the Department of Electronic and Electrical Engineering, University of Sheffield, S10 2TN, UK. E-mail: jiliang.zhang@sheffield.ac.uk.

Jie Zhang is with the Department of Electronic and Electrical Engineering, University of Sheffield, Sheffield, S10 2TN, UK, and also with Ranplan Wireless Network Design Ltd., Cambridge, CB23 3UY, UK.

This work was supported in part by the European Union's Horizon 2020 Research and Innovation Programme under Grant 766231 and Grant 752644. material's relative permittivity and thickness [11]. Measurement campaigns in the $28 \mathrm{GHz}$ band show that typical indoor building materials such as clear glass and dry wall usually have strong reflectivity and low penetration [12]. Hence, it is necessary to consider the effects of WR when evaluating indoor wireless performance.

In downlink multi-user (MU) MIMO scenarios, zero-forcing (ZF) precoding has been widely used to suppress inter-user interference. However, the power distribution across a $\mathrm{ZF}$ precoded transmit antenna array has not been sufficiently studied. The power assigned to each transmit antenna affects the efficiency of its corresponding RF power amplifier, and thus influences the power consumption of the RF chains of the AP [13]. It has been shown that the uniform power excitation over all transmit antennas would allow the RF power amplifiers to work with maximum efficiency [14], [15], while significant power variations across different antennas would reduce this efficiency and cause a huge waste of energy. That is why perantenna power constraints, instead of sum power constraints, need to be well considered for practical precoder design [16].

In this paper, we study the impact of WR on the perantenna power distribution across the precoded antenna array for indoor mmWave MU-MIMO downlink transmissions. The transmitter is assumed to be equipped with a ZF-precoded uniform linear array (ULA) for analytical tractability. The contribution of this paper is summarised as follows: 1) To capture the effects of WR on the power distribution across the precoded ULA, a new channel model characterising both the line-of-sight (LOS) path and the WR path is proposed for indoor MU-MIMO downlink scenarios. 2) We evaluate the effects of WR on the power distribution across the ZFprecoded ULA that serves multiple user equipment (UE) simultaneously through the comparisons between our proposed channel model and the pure LOS channel model in [15]. The unevenness in the power distribution across the ZF-precoded ULA becomes more significant under the effects of WR. 3) We analytically derive the entries of the ZF precoding matrix for a dual-UE case, the accuracy of which is verified by simulation results. 4) Our numerical results give insights into how the configuration of transmit antenna array, the spatial distribution of multiple users, the EM and physical properties of the wall, and the mutual coupling influence the power distribution across the ZF-precoded ULA.

\section{SySTEM MODEL}

In this work, we consider an indoor MU-MIMO downlink narrowband system in a LOS environment, as shown in Fig. 1(a). The AP, outfitted with a ULA of $M$ directional antennas, is deployed in parallel with a wall with a distance of $D_{1} \mathrm{~m}$ from the wall. The impact of the directional radiation pattern 

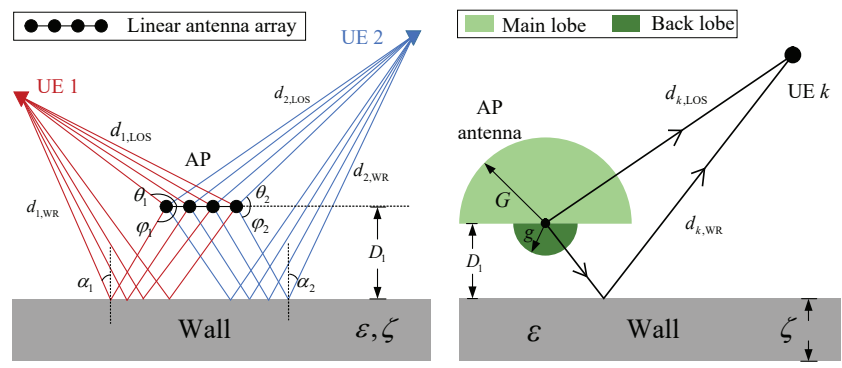

(a) The LOS path and the WR path (b) The directional radiation patbetween indoor BS and UE

Fig. 1. Indoor MU-MIMO system model for a LOS environment

of an AP antenna on the LOS path and the WR path is illustrated in Fig. 1(b). All the $K$ single-antenna UEs locate in the far-field of the AP's ULA. Due to the dynamic attitude of UEs, the UE antenna is assumed as an omnidirectional antenna for analytical tractability. The transmit ULA is assumed to be transparent, i.e., it will not block the WRs to the UEs.

In order to study the effects of WR on the per-antenna power distribution across the ULA, we incorporate the WR path to characterise the EM wave propagation inside a wall. The signal along the WR path experiences multiple reflections inside the wall closest to the AP, named as the considered wall hereafter. Using Friis' formula, the wireless link between the $m$ th antenna and the $k$ th UE is deterministically modelled as

$$
\begin{aligned}
& h_{k, m} \propto G d_{k, \mathrm{LOS}}^{-1} \exp \left(-j \beta\left(d_{k, \mathrm{LOS}}+\left(m-\frac{M+1}{2}\right) D \cos \theta_{k}\right)\right) \\
& +g \Gamma_{k} d_{k, \mathrm{WR}}^{-1} \exp \left(-j \beta\left(d_{k, \mathrm{WR}}+\left(m-\frac{M+1}{2}\right) D \cos \varphi_{k}\right)\right),
\end{aligned}
$$

where $\propto$ denotes being proportional to, $m \in\{1,2, \ldots, M\}$, $k \in\{1,2, \ldots, K\}, G$ and $g$ denote the main-lobe gain and sidelobe gain of each antenna, respectively, subject to $G^{2}+g^{2}=2$ according to energy conservation law, $d_{k, \text { LOS }}$ and $d_{k \text {,WR }}$ denote the length of the LOS path and the WR path of the $k$ th UE, respectively, $\theta_{k}$ and $\varphi_{k}$ denote the angle of departure (AOD) of the LOS path and the WR path of the $k$ th UE, respectively, $\beta=\frac{2 \pi}{\lambda}$ denotes the wave number with $\lambda$ being the wavelength, $D$ denotes the inter-antenna spacing, and $\Gamma_{k}$ denotes the $k$ th UE's equivalent reflection coefficient of multiple internal reflections along the WR path and is defined in the following.

Supposing that the considered wall is a homogenous dielectric reflector, the multiple internal reflections inside it are strongly affected by the first-order reflection. In this letter, we mainly focus on the transverse electric (TE) polarisation of the incident EM waves. The proposed approach can be directly applied for the transverse magnetic (TM) polarisation. The first-order reflection coefficient for UE $k$ is given by [11]

$$
\bar{\Gamma}_{k}=\frac{\cos \alpha_{k}-\sqrt{\varepsilon-\sin ^{2} \alpha_{k}}}{\cos \alpha_{k}+\sqrt{\varepsilon-\sin ^{2} \alpha_{k}}}
$$

with $\alpha_{k}=\left(\frac{\pi}{2}-\varphi_{k}\right)$ denoting the equivalent incident angle under plane wave assumption and $\varepsilon$ denoting the relative permittivity of the considered wall. Given the thickness of the considered wall as $\zeta$, the equivalent coefficient of multiple internal reflections for UE $k$ is given by

$$
\Gamma_{k}=\frac{1-\exp \left(-j 2 \tau_{k}\right)}{1-\bar{\Gamma}_{k}^{2} \exp \left(-j 2 \tau_{k}\right)} \bar{\Gamma}_{k}
$$

where $\tau_{k}=\frac{2 \pi \zeta}{\lambda} \sqrt{\varepsilon-\sin ^{2} \alpha_{k}}$.

After precoding at the ULA and propagating through a complex flat-fading channel, the received signals at the UE sides are given by

$$
\mathbf{y}=\mathbf{H C W} \mathbf{s}+\mathbf{n},
$$

where $\mathbf{y}, \mathbf{s}, \mathbf{n} \in \mathbb{C}^{K \times 1}$ denote the received signal vector, data symbol vector and additive white Gaussian noise vector, respectively, $\mathbf{H} \in \mathbb{C}^{K \times M}$ denotes the channel matrix whose elements are given in (1), $\mathbf{W} \in \mathbb{C}^{M \times K}$ denotes the precoding matrix, and $\mathbf{C} \in \mathbb{C}^{M \times M}$ denotes the mutual coupling matrix whose elements reveal how the radiated power of an antenna is affected by its neighbouring antennas [17].

In this letter, we employ the following empirical model of mutual coupling [18]

$$
\begin{aligned}
& C_{p, q}=\exp \left(-\frac{2 d_{p, q}}{\lambda}(\gamma+j \pi)\right), p \neq q \in\{1,2, \ldots, M\}, \\
& C_{p, p}=1-\frac{1}{M} \sum_{p=1}^{M} \sum_{q=1, q \neq p}^{M} C_{p, q},
\end{aligned}
$$

where $C_{p, q}$ and $d_{p, q}$, respectively, denote the mutual coupling coefficient and distance between the $p$ th antenna and $q$ th antenna, and $\gamma$ is a positive parameter controlling the mutual coupling level.

\section{EfFects of WALl Reflection ON THE Power Distribution OF ZF-PRECODED ULA}

In this section, we investigate how the power is distributed among the $M$ antennas of a ZF-precoded ULA serving $K$ UEs simultaneously. We first apply two metrics to evaluate the power distribution across the transmit precoded ULA for an arbitrary $K$ and then derive the analytical expression of the $\mathrm{ZF}$ precoding matrix for a dual-UE case, i.e. $K=2$.

\section{A. Metrics for quantifying power variation}

Considering uncorrelated and unit variance data symbol sequences $\mathbf{x}=\sum_{k=1}^{K} \mathbf{w}_{k} s_{k}$, the transmitted power of the AP's ULA is given by $\mathrm{E}\left(|\mathbf{x}|^{2}\right)=\sum_{k=1}^{K}\left\|\mathbf{w}_{k}\right\|^{2}$, where $\mathbf{w}_{k}$ and $s_{k}$ are the $M$-by-1 precoding vector and the intended symbol for the $k$ th UE, respectively, $\mathrm{E}(\cdot)$ denotes the expectation, and $\|\cdot\|^{2}$ denotes the F-norm. Since the transmitted power is the summation of the F-norm of each UE's precoding vector, the power distribution across the AP's ULA can be evaluated based on each UE's precoding vector. Following [15], we apply the two metrics below to evaluate the power imbalance among the transmit antennas.

The normalized power allocated to the $m$ th antenna to serve the $k$ th $\mathrm{UE}$ is defined as

$$
P_{k, m}^{\text {norm }}=\frac{\left|w_{k, m}\right|^{2}}{\left\|\mathbf{w}_{k}\right\|^{2}}=\frac{\left|w_{k, m}\right|^{2}}{\sum_{m=1}^{M}\left|w_{k, m}\right|^{2}},
$$

where $w_{k, m}$ is the $m$ th element of $\mathbf{w}_{k}$.

Maximum power ratio (MPR) describing the power variation across the $M$ antennas of a transmit ULA is defined as

$$
P_{k} \triangleq \frac{\max \left(\mathbf{P}_{k}^{\text {norm }}\right)}{\min \left(\mathbf{P}_{k}^{\text {norm }}\right)}
$$

where $\mathbf{P}_{k}^{\text {norm }}=\left(P_{k, 1}^{\text {norm }}, \ldots, P_{k, M}^{\text {norm }}\right)$ gives the normalized power levels allocated to the $M$ antennas for serving UE $k$. 


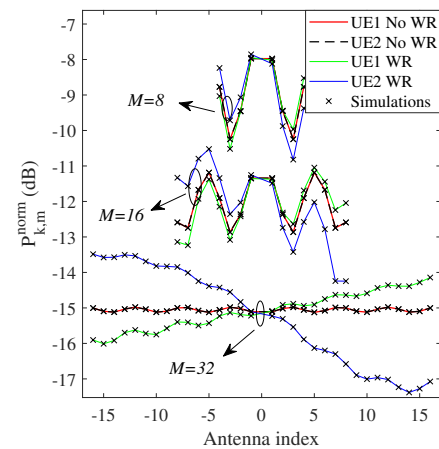

(a) Dual-UE case

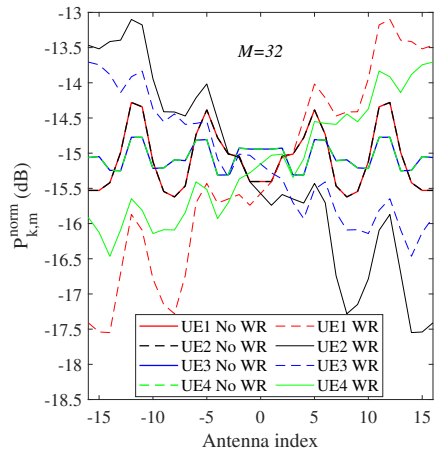

(b) Four-UE case

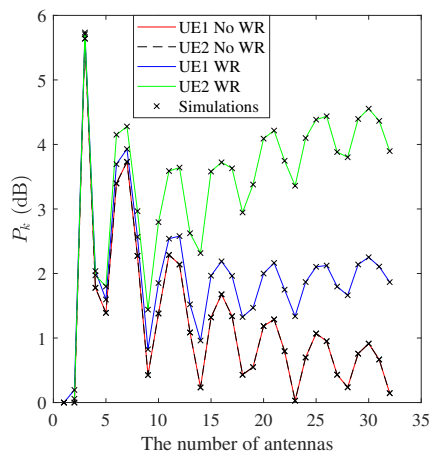

(c) Dual-UE case

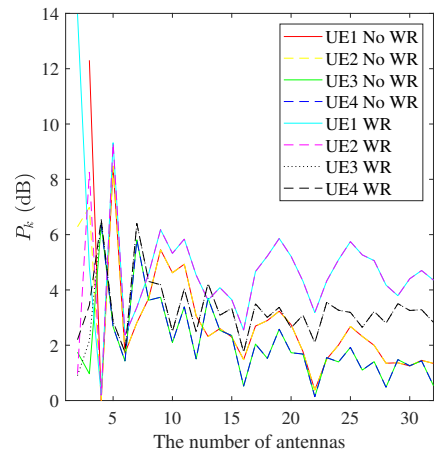

(d) Four-UE case

Fig. 2. The normalized power distribution and the MPR across the ZF-precoded ULA, where $D_{1}=0.05 \mathrm{~m}, \zeta=0.15 \mathrm{~m}, \gamma=5$, and $\varepsilon=5.31-j 0.3107$ for concrete [11]. Lines represent analytical results while markers represent simulation results. (Dual-UE case: $d_{1, \mathrm{LOS}}=6 \mathrm{~m}, \theta_{1}=\frac{5 \pi}{6}, d_{2, \mathrm{LOS}}=\sqrt{2} \mathrm{~m}$, $\theta_{2}=\frac{\pi}{4}$. Four-UE case: $d_{i, \mathrm{LOS}}=2 \mathrm{~m}$ for $i \in\{1,2,3,4\}, \theta_{1}=\frac{5 \pi}{6}, \theta_{2}=\frac{\pi}{6}, \theta_{3}=\frac{2 \pi}{3}, \theta_{2}=\frac{\pi}{3}$.)

\section{B. Analytical ZF precoding matrix for a dual-UE case}

The array factors (AFs) of the ULA are introduced as

$$
\begin{aligned}
\mathrm{AF}_{l l} & =\frac{\sin \left(M \beta D \delta_{l l} / 2\right)}{\sin \left(\beta D \delta_{l l} / 2\right)}, \\
\mathrm{AF}_{l k, n} & =\frac{\sin \left(M \beta D \delta_{l k, n} / 2\right)}{\sin \left(\beta D \delta_{l k, n} / 2\right)}
\end{aligned}
$$

where $k \neq l \in\{1,2\}$ denotes the UE index, $\delta_{l l}=\cos \theta_{l}-\cos \varphi_{l}$, $n \in\{3,4,5,6\}, \delta_{l k, 3}=\cos \theta_{k}-\cos \theta_{l}, \delta_{l k, 4}=\cos \varphi_{k}-\cos \theta_{l}$, $\delta_{l k, 5}=\cos \theta_{k}-\cos \varphi_{l}$, and $\delta_{l k, 6}=\cos \varphi_{k}-\cos \varphi_{l}$. Note that $\mathrm{AF}_{l k, n}=\mathrm{AF}_{k l, n}^{*}$, in which $\{\cdot\}^{*}$ denotes the complex conjugate.

Given (4), the effective downlink channel model is given by $\overline{\mathbf{H}}=\mathbf{H C}$. Even though $\mathbf{C}$ in (5) is a Toeplitz symmetrical matrix, the closed-form expression of $\mathbf{W}$ is still intractable. Fortunately, as stated in [19], the mutual coupling is nonnegligible only when $D$ is below $0.2 \lambda$. In this letter, $D$ is assumed to be half wavelength to guarantee a low spatial correlation [20]. As such, both $\mathbf{C} \approx \mathbf{I}$ and $\overline{\mathbf{H}} \approx \mathbf{H}$ are satisfied, especially when $\gamma \geq 4$.

When $\gamma \geq 4$, the ZF precoding matrix is derived as [21]

$$
\mathbf{W}=\mathbf{H}^{\mathrm{H}}\left(\mathbf{H H}^{\mathrm{H}}\right)^{-1} \propto \mathbf{H}^{\mathrm{H}}\left[\begin{array}{cc}
\left(\mathbf{H H}^{\mathrm{H}}\right)_{22} & -\left(\mathbf{H H}^{\mathrm{H}}\right)_{12} \\
-\left(\mathbf{H H}^{\mathrm{H}}\right)_{21} & \left(\mathbf{H} \mathbf{H}^{\mathrm{H}}\right)_{11}
\end{array}\right]_{10}
$$

where $\{\cdot\}^{\mathrm{H}}$ represents the complex conjugate transpose of a matrix. The four elements of matrix $\mathbf{H} \mathbf{H}^{\mathrm{H}}$ are given by (11)(14) at the bottom of this page, where

$$
\begin{aligned}
F_{l} & =\exp \left(j \beta\left(d_{l, \mathrm{LOS}}-d_{l, \mathrm{WR}}\right)\right) \mathrm{AF}_{l l}, l \in\{1,2\}, \\
F_{21,3} & =\exp \left(j \beta\left(d_{1, \mathrm{LOS}}-d_{2, \mathrm{LOS}}\right)\right) \mathrm{AF}_{21,3}, \\
F_{21,4} & =\exp \left(j \beta\left(d_{1, \mathrm{WR}}-d_{2, \mathrm{LOS}}\right)\right) \mathrm{AF}_{21,4}, \\
F_{21,5} & =\exp \left(j \beta\left(d_{1, \mathrm{LOS}}-d_{2, \mathrm{WR}}\right)\right) \mathrm{AF}_{21,5}, \\
F_{21,6} & =\exp \left(j \beta\left(d_{1, \mathrm{WR}}-d_{2, \mathrm{WR}}\right)\right) \mathrm{AF}_{21,6} .
\end{aligned}
$$

Note that $F_{l k, n}=F_{k l, n}^{*}$ for $n \in\{3,4,5,6\}$ and $k \neq l \in\{1,2\}$.

By substituting (11)-(14) into (10), the precoding coefficient for the $m$ th antenna to serve the $k$ th $\mathrm{UE}$ is derived as

$$
w_{k, m} \propto h_{k, m}^{*}\left(\mathbf{H H}^{\mathrm{H}}\right)_{l l}-h_{l, m}^{*}\left(\mathbf{H H}^{\mathrm{H}}\right)_{l k}, k \neq l \in\{1,2\} .
$$

When $\gamma<4$, since the analytical expression of $\mathbf{W}$ is not tractable, we analyse the proposed metrics using simulations.

\section{NuMERICAL RESULTS}

In order to study how WR affects the power distribution across the ZF-precoded ULA in the case of the AP being deployed close to the considered wall, we compare our proposed channel model in (1)-(3) with the LOS channel model in [15], which is given as

$$
h_{k, m}^{\prime} \propto d_{k, \mathrm{LOS}}^{-1} \exp \left(-j \beta\left(d_{k, \mathrm{LOS}}+\left(m-\frac{M+1}{2}\right) D \cos \theta_{k}\right)\right)
$$

for $m \in\{1,2, \ldots, M\}$ and $k \in\{1,2, \ldots, K\}$. As the channel model in [15] considers only the LOS path without the WR path, it is referred to as the pure LOS case. The normalized power $P_{k, m}^{\text {norm }}$ and MPR $P_{k}$, given in (6) and (7) respectively, are taken as metrics for the comparison.

The simulation is carried out at $28 \mathrm{GHz}$. The inter-antenna spacing of the ULA is half wavelength. The main-lobe gain and side-lobe gain of the radiation pattern is assumed as $G=\sqrt{5 / 3}$ and $g=\sqrt{1 / 3}$, respectively. The incident EM wave impinging on the wall surface is assumed to be TE polarised. All the antennas of the AP and UEs are assumed to be vertically polarised (that is, there is no polarisation mismatch). The location of UE $k$ is specified with the length and the AOD of its LOS path from the AP, i.e. $d_{k, \text { LOS }}$ and $\theta_{k}$. Note that the relative permittivity of a wall material is a complex value, whose real part and imaginary part are functions of the carrier

$$
\begin{gathered}
{\left[\mathbf{H H}^{\mathrm{H}}\right]_{11}=M\left(G^{2} d_{1, \mathrm{LOS}}^{-2}+g^{2} d_{1, \mathrm{WR}}^{-2}\left|\Gamma_{1}\right|^{2}\right)+2 G g d_{1, \mathrm{WR}}^{-1} d_{1, \mathrm{LOS}}^{-1} \operatorname{Re}\left(\Gamma_{1} F_{1}\right)} \\
{\left[\mathbf{H H}^{\mathrm{H}}\right]_{22}=M\left(G^{2} d_{2, \mathrm{LOS}}^{-2}+g^{2} d_{2, \mathrm{WR}}^{-2}\left|\Gamma_{2}\right|^{2}\right)+2 G g d_{2, \mathrm{WR}}^{-1} d_{2, \mathrm{LOS}}^{-1} \operatorname{Re}\left(\Gamma_{2} F_{2}\right)} \\
{\left[\mathbf{H H}^{\mathrm{H}}\right]_{12}=G^{2} d_{2, \mathrm{LOS}}^{-1} d_{1, \mathrm{LOS}}^{-1} F_{12,3}+G g d_{2, \mathrm{LOS}}^{-1} d_{1, \mathrm{WR}}^{-1} \Gamma_{1} F_{12,4}+G g d_{1, \mathrm{LOS}}^{-1} d_{2, \mathrm{WR}}^{-1} \Gamma_{2}^{*} F_{12,5}+g^{2} d_{1, \mathrm{WR}}^{-1} d_{2, \mathrm{WR}}^{-1} \Gamma_{1} \Gamma_{2}^{*} F_{12,6}} \\
{\left[\mathbf{H H}^{\mathrm{H}}\right]_{21}=G^{2} d_{2, \mathrm{LOS}}^{-1} d_{1, \mathrm{LOS}}^{-1} F_{21,3}+G g d_{2, \mathrm{LOS}}^{-1} d_{1, \mathrm{WR}}^{-1} \Gamma_{1}^{*} F_{21,4}+G g d_{1, \mathrm{LOS}}^{-1} d_{2, \mathrm{WR}}^{-1} \Gamma_{2} F_{21,5}+g^{2} d_{1, \mathrm{WR}}^{-1} d_{2, \mathrm{WR}}^{-1} \Gamma_{1}^{*} \Gamma_{2} F_{21,6}}
\end{gathered}
$$




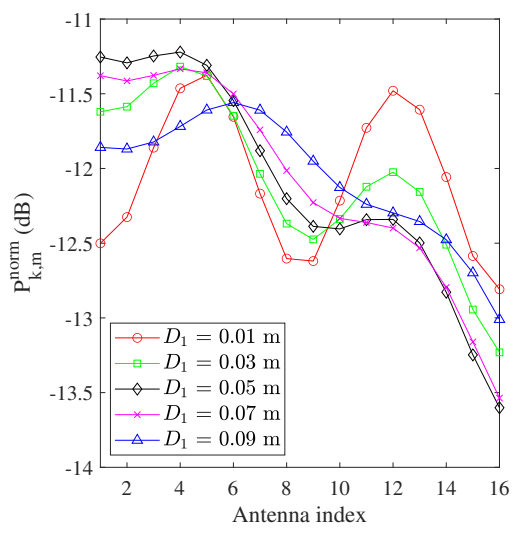

(a) The normalized power distribution for different AP-wall distances where $d_{1, \mathrm{LOS}}=d_{2, \mathrm{LOS}}=2 \mathrm{~m}, \theta_{1}=\frac{\pi}{6}, \theta_{2}=\frac{5 \pi}{6}$.

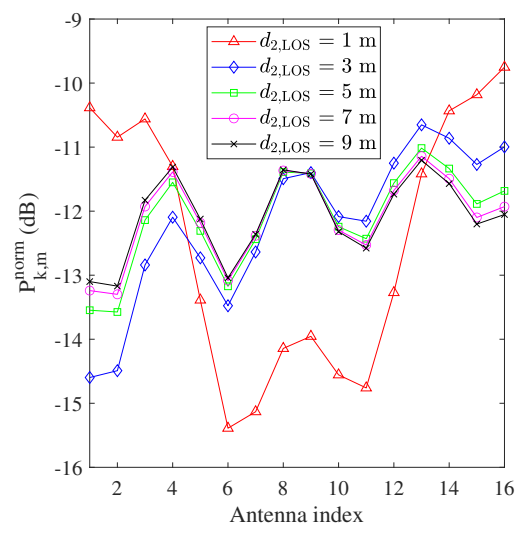

(b) The normalized power distribution for different values of $d_{2, \text { LOS }}$ where $d_{1, \mathrm{LOS}}=\sqrt{2}$ $\mathrm{m}, \theta_{1}=\frac{\pi}{4}, \theta_{2}=\frac{5 \pi}{6}, D_{1}=0.05 \mathrm{~m}$.

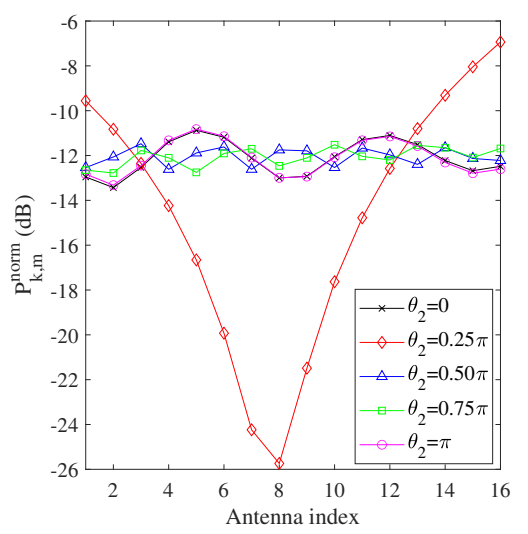

(c) The normalized power distribution for different values of $\theta_{2}$ where $d_{2, \mathrm{LOS}}=6 \mathrm{~m}$, $d_{1, \mathrm{LOS}}=\sqrt{2} \mathrm{~m}, \theta_{1}=\frac{\pi}{4}, D_{1}=0.05 \mathrm{~m}$.

Fig. 3. The normalized power distribution across the ZF-precoded ULA under different AP-wall distances and user spatial distributions, where $M=16$, $\zeta=0.15 \mathrm{~m}, \gamma=5$, and $\varepsilon=5.31-j 0.3107$ for concrete.

frequency, respectively [11]. The following simulation results can verify the analytical derivations in Section III-B.

Fig. 2(a) plots the normalized power distribution across the ZF-precoded ULA with or without considering WR for the dual-UE case, for the number of antennas being 8,16 , or 32 . We can see that the normalized power distribution presents a periodic behaviour across the antennas when considering only the LOS path. However, the vibration in power distribution caused by the WR breaks this periodicity.

Fig. 2(b) plots the normalized power distribution across the ZF-precoded ULA with or without considering WR for the four-UE case for the number of antennas being 32. Comparing the results obtained using the two channel models, we observe that the effects of WR exacerbates the power variation up to 6 $\mathrm{dB}$ across the ZF-precoded ULA, revealing that some antennas are allocated much more power than the others.

Fig. 2(c) and Fig. 2(d) show the impact of the number of antennas on the MPR across the ZF-precoded ULA with or without considering WR for the dual-UE case and the fourUE case, respectively. We can see that the MPRs obtained by our proposed channel model are larger than that obtained by the pure LOS channel model, indicating that the effects of WR exacerbates the uneven power distribution across the ZF-precoded ULA. For the dual-UE case under the pure LOS channel, MPR becomes smaller gradually when the number of antennas increases. However, under our proposed channel, increasing the number of antennas would exacerbate the nonuniform power distribution over the ULA.

Fig. 3(a) illustrates the normalized power distribution of the ZF-precoded ULA for UE 2 under different AP-wall distances for a dual-UE case, where the positions of UE 1 and UE 2 are assumed to be symmetric with respect to the midpoint of the ULA for simplicity. The result of UE 1 is symmetrical to that of UE 2 with respect to the midpoint of ULA. As shown in Fig. 3(a), the variation in the power distribution across the ULA changes greatly with the AP-wall distance, which reveals that the AP-wall distance has to be taken into account carefully.

Fig. 3(b) and Fig. 3(c) present the normalized power dis- tribution of the ZF-precoded ULA for different values of the length (from 1 to $9 \mathrm{~m}$ ) and the $\mathrm{AoD}$ (from 0 to $\pi$ ) of the LOS path of one user in the dual-UE case, respectively. Fig. 3(b) shows that, as the length of the LOS path increases, the normalized power for serving the UE varies slower within a smaller dynamic range, which is also observed in Fig. 2(a). In Fig. 3(c), a significant variation appears in the power distribution across the ZF-precoded ULA when $\theta_{2}$ approaches $\pi / 4$. This is ascribed to the location of UE 2 being very close to UE 1 with $\theta_{1}=\pi / 4$. In this case, the ZF precoder needs to allocate highly different power levels across the ULA to distinguish the two UEs.

From Fig. 3(b)-(c) and Fig. 2(a)-(d), we can see that the power distribution is not sensitive to the length (UE-AP distance) or the AoD of the LOS path under the pure LOS channel model, while under our proposed channel model, different multi-user spatial distributions result in different dynamic ranges of the power distribution over the ULA, which is more evident when the UE is closer to the wall.

In Fig. 4(a)-(c), the two UEs are assumed to be symmetrically located with respect to the midpoint of the ULA for simplicity and only the result of UE 2 is plotted. The result of UE 1 is symmetrical to that of UE 2 with respect to the midpoint of ULA.

Fig. 4(a) and Fig. 4(b) present the normalized power distribution across the ZF-precoded ULA for different values of the real part of wall permittivity (from 1 to 9 ) and wall thickness (from 0.1 to $0.3 \mathrm{~m}$ ) for the dual-UE case, respectively. We can see from Fig. 4(a) that the non-uniform power distribution across the ZF-precoded ULA will be exaggerated with the increase of the real part of wall permittivity. Differences as large as $8 \mathrm{~dB}$ are observed between the ends of the ULA. In Fig. 4(b), the power distribution does not change with the wall thickness. This is because the wavelength at $28 \mathrm{GHz}$, i.e. 1 $\mathrm{cm}$, is not comparable to the typical wall thickness.

Fig. 4(c) plots the normalized power distribution across the ZF-precoded ULA for different values of $\gamma$. We can see that the power distribution across the ZF-precoded ULA changes 


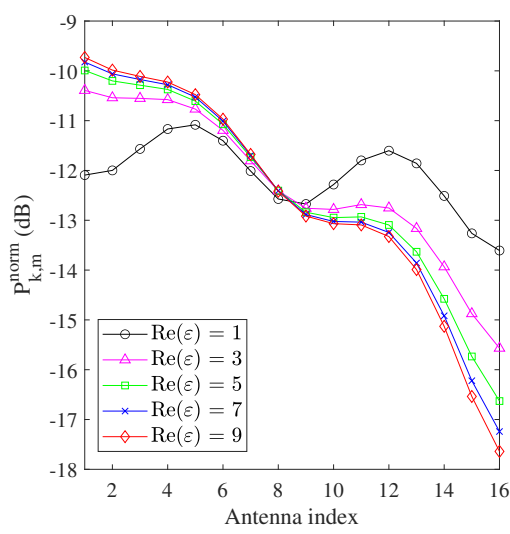

(a) The normalized power distribution under different wall permittivities where $\gamma=5$, $\zeta=0.15 \mathrm{~m}$.

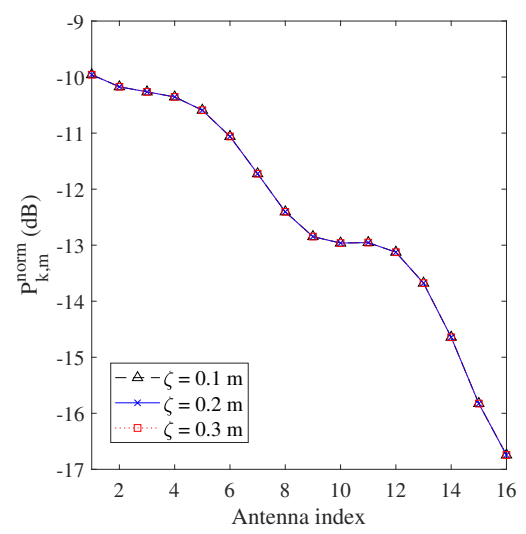

(b) The normalized power distribution for different values of wall thickness where $\gamma=5$, and $\varepsilon=5.31-j 0.3107$ for concrete.

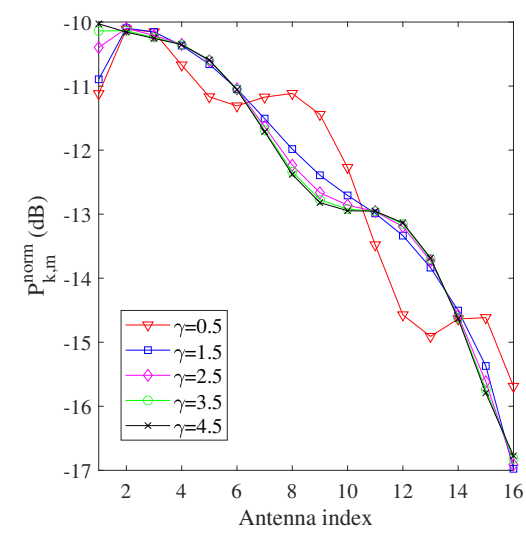

(c) The normalized power distribution for different values of $\gamma$ where $\zeta=0.15 \mathrm{~m}$, and $\varepsilon=5.31-j 0.3107$ for concrete.

Fig. 4. The impact of wall permittivity, thickness, and mutual coupling on the normalized power distribution across the ZF-precoded ULA, where $d_{1, \mathrm{LOS}}=$ $d_{2, \text { LOS }}=2 \mathrm{~m}, \theta_{1}=\frac{\pi}{6}, \theta_{2}=\frac{5 \pi}{6}, M=16, D_{1}=0.05 \mathrm{~m}$.

slightly with $\gamma$ ranging from 1.5 to 4.5 , corresponding to the mutual coupling level between two adjacent antennas from $13.03 \mathrm{~dB}$ to $-39.09 \mathrm{~dB}$ [18]. However, for a very small value of $\gamma$, e.g., $\gamma=0.5$ corresponding to the mutual coupling level between two adjacent antennas of $-4.34 \mathrm{~dB}$, the mutual coupling has to be considered while analysing the power distribution over the AP's ULA.

\section{CONCLUSION AND FUTURE WORKS}

In this paper, we build an indoor MU-MIMO downlink channel model that involves both the LOS path and the WR path. The expression of the ZF precoding matrix for the dualUE case is analytically derived. For the scenario where the AP is deployed close to a wall, we compare the power distribution across the ULA under our channel model and that under the pure LOS channel model, in terms of the normalized power distribution and MPR. Numerical results show that the power distribution becomes more uneven across the ZF-precoded ULA under the effects of WR. Even though the proposed numerical approach has shed light on how WR impacts the per-antenna power distribution, a comprehensive measurement campaign is still required to verify the results of this work.

The following research will be considered in future works: 1) Experimental validation of the proposed approach; 2) Consideration of actual antenna patterns; 3 ) The impact of an arbitrary arrangement of the AP; 4) The effects of WR on the power distribution across an MMSE/MRT-precoded massive MIMO antenna array; 5) Indoor rich scattering environment; 6) MmWave wideband wireless system.

\section{REFERENCES}

[1] J. Kibiłda, et al., "Indoor millimeter-wave systems: design and performance evaluation," Proceedings of the IEEE, Apr. 2020

[2] V. Raghavan, et al., "Millimeter-wave MIMO prototype: measurements and experimental results," IEEE Commun. Mag., vol. 56, no. 1, pp. 202209, Jan. 2018.

[3] E. Torkildson, et al., "Indoor millimeter wave MIMO: feasibility and performance," IEEE Trans. Wireless Commun., vol. 10, no. 12, pp. 41504160, Dec 2011.
[4] E. Torkildson,et al., "Channel modeling for millimeter wave MIMO," Information Theory and Applications Workshop (ITA), 2010, pp. 1-8.

[5] E. Torkildson, et al., "Millimeter-wave spatial multiplexing in an indoor environment," IEEE Globecom Workshops, 2009, pp. 1-6.

[6] J. Zhang and G. De La Roche, "Femtocells: technologies and deployment," Wiley, 2010.

[7] S. K. Yoo, et al., "Ceiling- or wall-mounted access points: an experimental evaluation for indoor millimeter wave communications," Proc. IEEE 13th Eur. Conf. Antennas Propag. (EUCAP), 2019, pp. 1-5.

[8] Y. Zhang, et al., "How friendly are building materials as reflectors to indoor LOS MIMO communications?," IEEE Internet Things J., early access, 2020.

[9] J. Zhang, et al., "Wireless energy efficiency evaluation for buildings under design based on analysis of interference gain," IEEE Trans. Veh. Tech., vol. 69, no. 6, pp. 6310-6324, Jun. 2020.

[10] J. Zhang, et al., "Two-ray reflection resolution algorithm for planar material electromagnetic property measurement at the millimeter-wave bands," Radio Sci., , vol. 55, no. 3, Mar. 2020.

[11] "Effects of building materials and structures on radiowave propagation above about $100 \mathrm{MHz} \mathrm{P}$ series radiowave propagation," IETF, ITURecommendation P.2040-1, Jul. 2015.

[12] H. Zhao, et al., " $28 \mathrm{GHz}$ millimeter wave cellular communication measurements for reflection and penetration loss in and around buildings in New York city", Proc. IEEE Int. Conf. Commun. (ICC), 2013, pp. 5163-5167.

[13] P. Skrimponis, et al., "Power consumption analysis for mobile mmWave and sub-THz receivers," 2020 2nd $6 G$ Wireless Summit (6G SUMMIT), Levi, Finland, 2020, pp. 1-5.

[14] P. Angeletti and G. Toso, "Array antennas with jointly optimized elements positions and dimensions Part I: linear arrays," IEEE Trans. Antennas Propag., vol. 62, no. 4, pp. 1619-1626, Apr. 2014.

[15] N. Amani, et al., "Per-antenna power distribution of a zero-forcing beamformed ULA in pure LOS MU-MIMO," IEEE Commun. Lett., vol. 22, no. 12, pp. 2515-2518, Dec. 2018.

[16] T. M. Pham, et al., "Efficient zero-forcing precoder design for weighted sum-rate maximization with per-antenna power constraint," IEEE Trans. Veh. Technol., vol. 67, no. 4, pp. 3640-3645, Apr. 2018.

[17] M. Gustafsson, et al., "Impact of mutual coupling on capacity in large MIMO antenna arrays," Proc. IEEE 13th Eur. Conf. Antennas Propag. (EUCAP), 2014, pp. 2723-2727.

[18] L. Savy and M. Lesturgie, "Coupling effects in MIMO phased array," IEEE Radar Conference (RadarConf), Philadelphia, PA, 2016, pp. 1-6

[19] X. Gao, et al., "Massive MIMO performance evaluation based on measured propagation data," IEEE Trans. Wirel. Commun., vol. 14, no. 7, pp. 3899-3911, Jul. 2015.

[20] D. S. Shiu, et al., "Fading correlation and its effect on the capacity of multielement antenna systems," IEEE Trans. Commun., vol. 48, no. 3, pp. 502-513, Mar. 2000.

[21] A. Wiesel, Y. C. Eldar and S. Shamai, "Zero-forcing precoding and generalized inverses," IEEE Trans. Signal Processing, vol. 56, no. 9, pp. 4409-4418, Sep. 2008. 